\title{
Morphological characteristics of chicken liver influenced by immunostimulants
}

\author{
Rezilya Asrutdinova, Nadezhda Kanalina, Lenar Rakhmatov, Rustem Mullakhmetov \\ Kazan State Academy of Veterinary Medicine named after N.E. Bauman, Kazan, 420029, Russia
}

\begin{abstract}
The paper studies the influence of Raspol polysaccharide and fosprenil on chicken liver microstructure during vaccination against avian infectious bronchitis. The response to the integrated effects of the vaccine and immunostimulants during this period caused weakly expressed edema in perisinusoid spaces and marked activation of reticuloendothelium cells represented by the increase of Kupffer's cells. On the $28^{\text {th }}$ day after vaccination, all hepatocytes were distinguished by uniformity of magnitude and shape. They lacked evidence of prior dystrophic changes. Most hepatocytes represented actively synthesizing cells as indicated by dark oxyphilous color of the cytoplasm. RES cells in the walls of sinus capillaries were still numerous, with a large volume of cytoplasm and nucleus. The previous studies refer to the phenomenon in the form of a cluster of lymphoid cells in the perivascular regions of the triad area, mucoid degeneration of vascular walls disappeared. The lumens of biliary passage were well observed, especially in chickens of the first group. The liver of the chicks of the control group after vaccination revealed weakly expressed local vascular disorders and absence of signs of metabolic disorders. Organ parenchyma cells retained structural signs of moderate biosynthetic activity of the cytoplasm and nucleus.
\end{abstract}

\section{Introduction}

Currently, world and domestic poultry farming is the fastest growing branch of the agro-industrial complex providing the population with high-quality animal products. Large poultry industrial enterprises, where the results of research work are effectively introduced, serve the basis of poultry farming in Russia [1].

For more than half a century the poultry industry has been providing the population with high-quality dietary products - poultry meat and egg, the production of which per capita grows from year to year [2].

As a rule, the intensification of the metabolic processes of the bird's body leads to the suppression of the components of the immune system, therefore, causes the need for its correction [3-8].

The judgment on the safety of immunomodulators is currently based on toxicity studies conducted in laboratory and other animals [9-12].

Under conditions of industrial poultry maintenance at high level of technological stress, secondary immunodeficiency states are recorded in almost $80 \%$ of poultry population [13-15]. Vaccination of poultry with low level of resistance does not lead to the production of sufficient number of protective antibodies and is accompanied by the increase of some postvaccinal complications $[16,17]$.

Polysaccharides are considered as a promising biocomplex for the creation of new drugs to correct various immune system disorders [18-20].
Given the above, the search for new immunostimulating drugs of domestic production is an urgent task that requires their study, testing and introduction into veterinary practice.

\section{Materials and Methods}

The efficiency of Raspol heteropolysaccharide was determined by immunizing chickens against infectious chicken bronchitis with the SEVAC IBird vaccine. This required 75 egg chickens of Lomann LSL cross, divided into 3 groups of 25 chickens each. The birds of the first group were given Raspol twice at a dose of $133.2 \mathrm{mg} / \mathrm{kg}$ with an interval of 3 days. The first administration on the day of vaccination, the second - 3 days after immunization; the second group was administered Fosprenyl at a dose of $0.05 \mathrm{ml} / \mathrm{kg}$ according to the same regimen; the control included 25 birds vaccinated against avian infectious bronchitis without an immunostimulant.

To study the liver microstructure, $0.5 \times 1.5 \times 0.5 \mathrm{~cm}$ samples were taken after slaughter. The material was fixed in a $10 \%$ aqueous solution of neutral formalin for three days. Fixed pieces of organs were dehydrated and treated with chloroform. Liver pieces dehydrated in ascending-density alcohols were compacted by pouring into paraffin. From the paraffin blocks paraffin sections were prepared on the sledge microtome and glued to the slide. After removal of paraffin residues, the sections were clarified with carbolic xylene and treated with balsam. The agents were stained with haematoxylin-

\footnotetext{
* Corresponding author: roza509a@mail.ru
} 
eosin. When studying microstructural changes in the internal organs and tissues of chickens, the most pronounced changes were photographed with Pentax camera using MBI-6 microscope.

\section{Results}

On the $7^{\text {th }}$ day after vaccination against avian infectious bronchitis the histological structure of liver (Fig. 1) with the use of Raspol as an immunostimulant was the same, relatively short rods were formed from homogeneous parenchyma cells.

The response to the integrated effects of the vaccine and immunostimulants during this period caused weakly expressed edema in perisinusoid spaces and marked activation of reticuloendothelium cells represented by the increase of Kupffer's cells. On the $14^{\text {th }}$ day of the experiment, the lumens of the sinus capillaries were moderately expanded with relatively large reticuloendothelian cells.

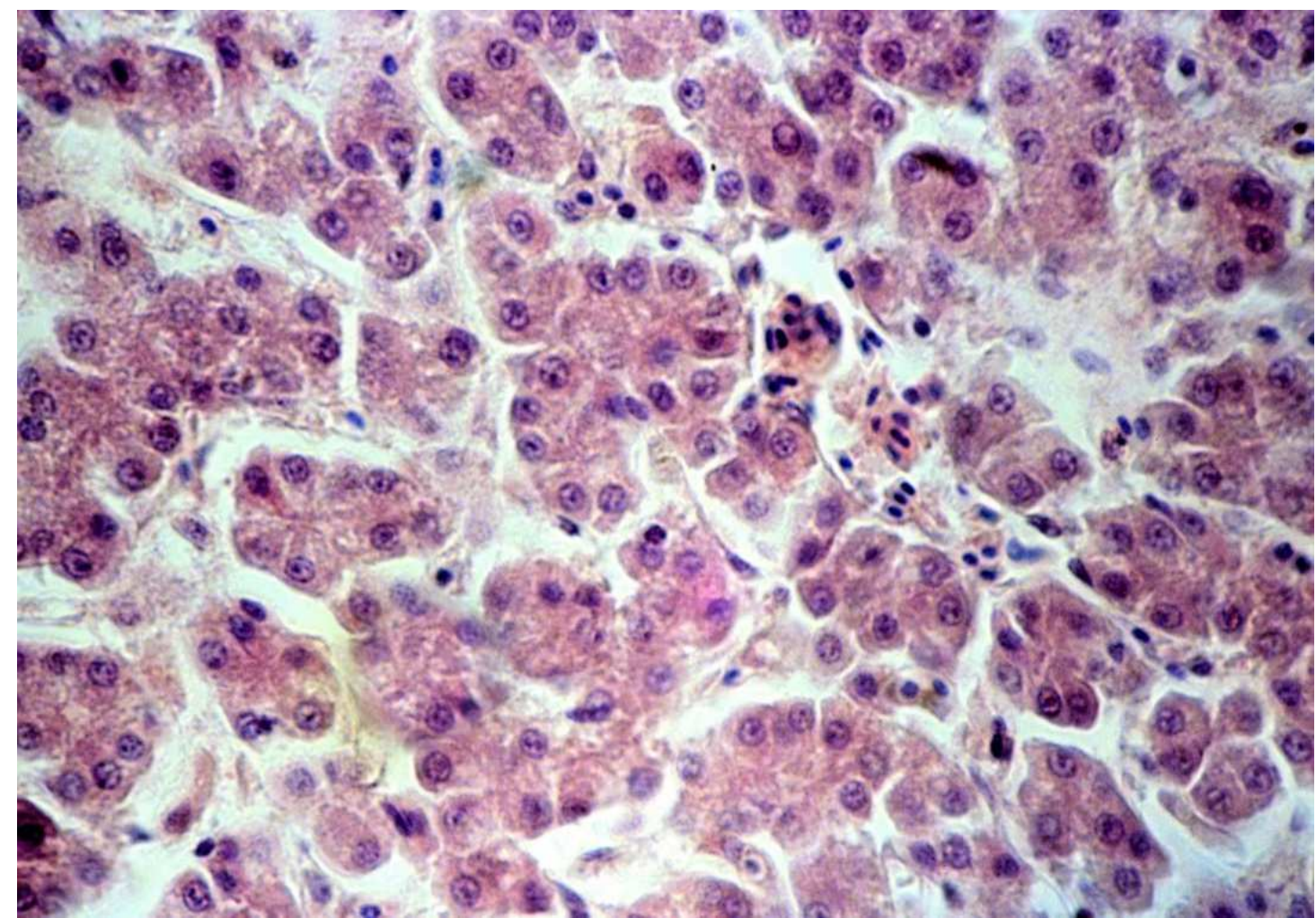

Fig. 1. Histology of chicken liver on the $7^{\text {th }}$ day after vaccination with Raspol as an immunostimulant. Coloring with hematoxylin and eosin. Image magnification x 400 .

Perisinusoid spaces were not indicated. Poultry liver for this postvaccinal period was distinguished by the absence of metabolic disorders and intra-organ hemocirculation. On the $21^{\text {st }}$ day after vaccination, the organ rods became less winding, not uniform in thickness. Hepatocytes differed in size and shape with a predominance of small-sized cytoplasm cells.

In the triad area, as a result of postvaccinal restructuring, small clusters of lymphoid cells were noticeable. During this period, as a result of vaccination changes, the expansion of the lumen of the central veins of sinusoid capillaries was noted in the absence of signs of swelling of perisinusoid spaces. By this time, stellate reticuloendotheliocytuses increased in volume were well distinguished.

The liver of chickens on the $28^{\text {th }}$ day after vaccination with the use of Raspol as an immunostimulant (Fig. 2) was characterized by moderate blood filling of sinusoid interchamber hemocapillars, the location of numerous noticeably reduced reticuloendothelium cells on their walls and the lack of marked perisinusoid spaces.
The histological structure of the chicken liver presented in Fig. 3, on the $7^{\text {th }}$ day after vaccination with the use of Fosprenyl as an immunostimulant shows sinusoid hemocapillars of weak blood filling. Small RES cells in them were distinguished by a small volume of nucleus and cytoplasm. The venous vessels of the organ were moderately dilated. In some sections of the liver, relatively large hepatocytes with dark oxyphilic color of the cytoplasm and with rounded-oval nuclei and the central location of large nuclei were found, reflecting the increased synthetic activity of these cells.

On the $14^{\text {th }}$ day after vaccination of rearing young chickens against avian infectious bronchitis in combination with Fosprenil, blood filling of the venous vessels of the organ markedly increased. The MPS cells that became more numerous increased markedly, their cytoplasm was noticeably rounded. As a reflection of the cellular response to the vaccine the clusters of lymphoid cells were detected around the vessels of triads. On the $21^{\text {st }}$ day after vaccination, in combination with the immunostimulant, the volume of MPS cells increased markedly. 
In some areas around the gathering veins of the liver, vessels of triads, the content of lymphoid cells increased, which was a reflection of the morphological equivalent of the delayed hypersensitivity reaction. On the $28^{\text {th }}$ day after vaccination (Fig. 4), all hepatocytes were isolated by uniformity of size and shape.

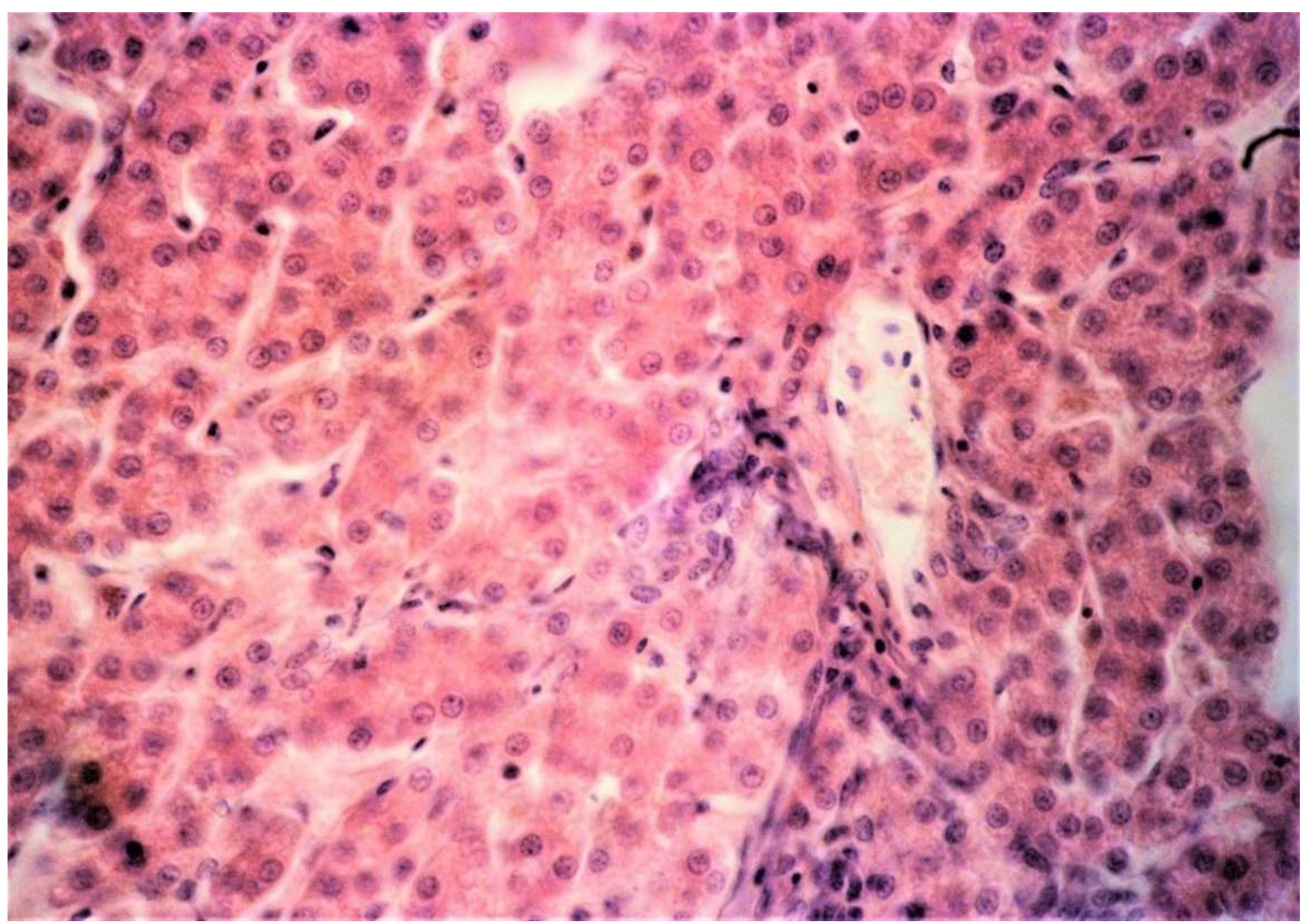

Fig. 2. Histology of chicken liver on the $28^{\text {th }}$ day after vaccination with application of Raspol as immunostimulant. Coloring with hematoxylin and eosin. Image magnification x 400 .

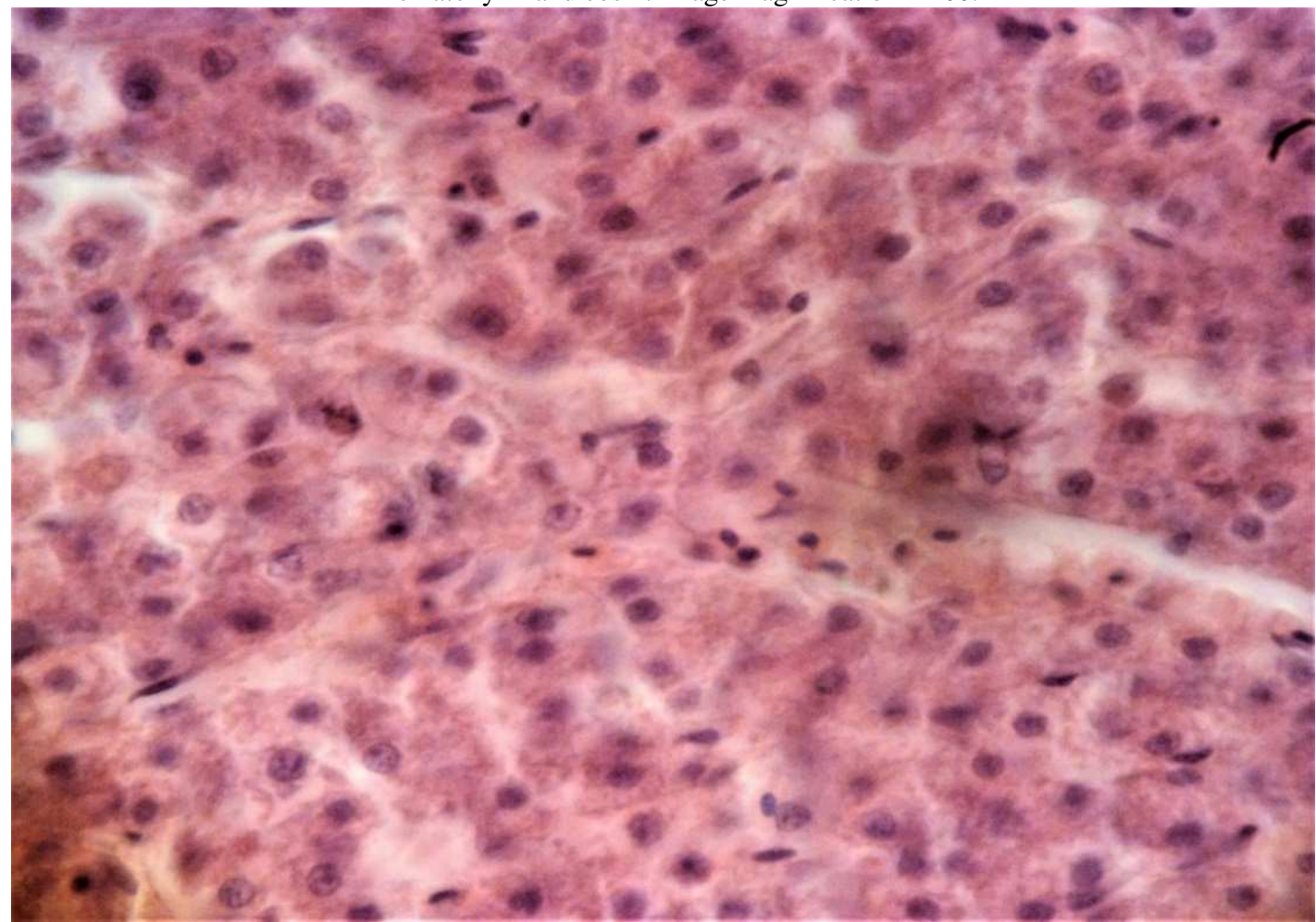

Fig. 3. Histology of chicken liver on the $7^{\text {th }}$ day after vaccination using Fosprenyl as an immunostimulant. Coloring with hematoxylin and eosin. Image magnification x 400 . 


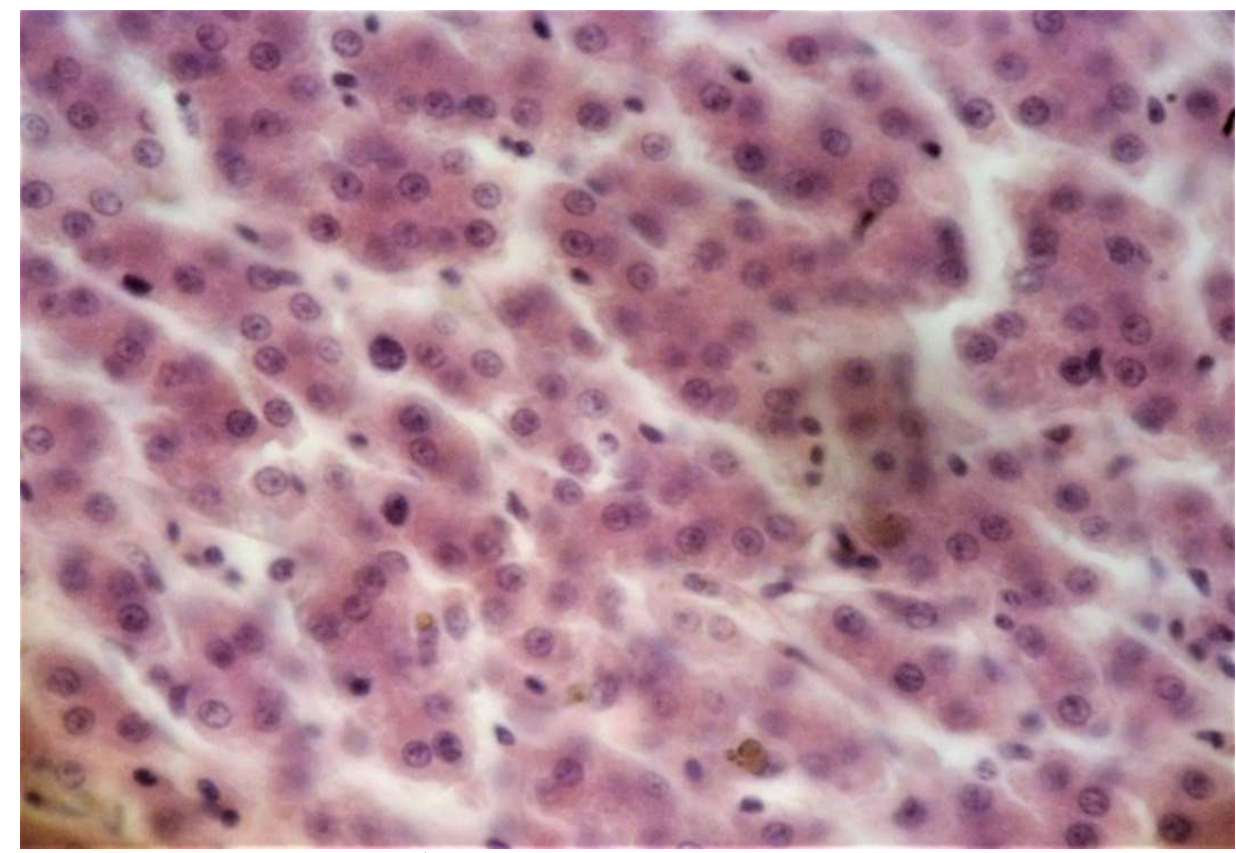

Fig. 4. Histology of chicken liver on the $28^{\text {th }}$ day after vaccination using Fosprenyl as an immunostimulant. Coloring with hematoxylin and eosin. Image magnification x 400 .

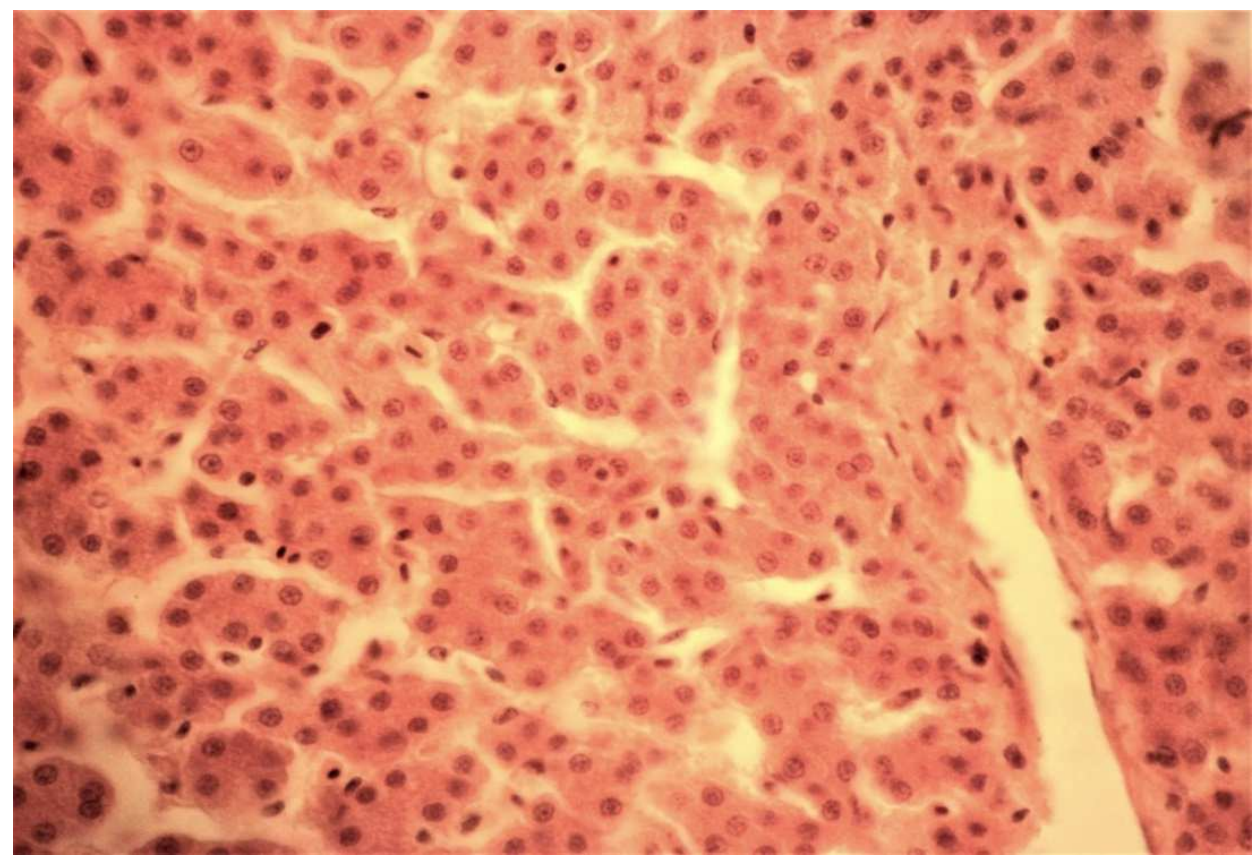

Fig. 5. Histology of chicken liver of the control group on the $28^{\text {th }}$ day after vaccination. Coloring with hematoxylin and eosin. Image magnification x 400 .

The histology of the liver of the control group after vaccination was weak. Throughout the area of the organ cut, focal and diffuse areas were noted with violation of the rod structure of the organ. The centers of rod discomplexation represented clusters of hepatocytes with poorly marked cytomembrane, a pale oxyphilous color of the cytoplasm and a small volume and rounded nuclei depleted of chromatin and the presence of extremely small, barely marked nuclei. Sinusoid hemocapillars were of mild blood filling. Few small stellate reticuloendotheliocytes were isolated with small hyperchromic nuclei. These cells were located unevenly on the surface of sinusoid capillaries. Hepatocytes with two nuclei were extremely rare. The changes noted in the organ parenchyma reflected a noticeable increase in the processes of metabolic disorders that do not go beyond reversible changes. On the $21^{\text {st }}$ day after vaccination, hepatocytes of the liver of the chickens of the control group acquired a structure uniform throughout the entire cut area. They have a light oxyphilous color of the cytoplasm with a well-defined cytomembrane. Uniform in size, rounded nuclei were distinguished by a larger number of nuclei located in the center or near the karyolemma. In some places there were a compact accumulation of hepatocytes with dark oxyphilous color of the cytoplasm. Sinusoidal capillaries were 
distinguished by a uniform moderate expansion of lumen profiles and the absence of designation of perisinusoid spaces.

Compared to the previous study period, the severity of hepatocyte protein dystrophy was noticeably reduced, and it bore the form of a cluster in a swollen cytoplasm of small oxyphilous stained grains. Most hepatocytes on the $28^{\text {th }}$ day after vaccination (Fig. 5) were marked by intense oxyphilous color of the cytoplasm and its uniform size. Most hepatocyte nuclei had a rounded shape and one or two nuclei centrally located. Compared to the previous study period, the presence of lymphoid cells increased in the area of triads, the profiles of the lumens of blood vessels were poorly indicated due to the preservation of moderate signs of mucoid degeneration.

Therefore, by this time, the manifestations of local vascular disorders and protein metabolism disorders in parenchyma cells were noticeably weakened in the liver. Thus, on the $28^{\text {th }}$ day previous disorders of local hemocirculation and metabolism became weaker.

\section{Conclusion}

Histomorphological studies of chicken liver sections indicate the stimulation of immune response with Raspol polysaccharide and fosprenil when vaccinating birds against avian infectious bronchitis. The most pronounced immunostimulation was noted in the first experimental group using Raspol.

\section{References}

1. V.I. Fisinin, N.A. Zhuravel, A.V. Miftakhutdinov, Veterinary Science. Methodology for determining the effectiveness of new veterinary methods and remedies in poultry farming, $\mathbf{6 , 1} 14$ (2018).

2. G.A. Bobyleva, Directions determining the development of poultry farming in the near future, Poultry and poultry products, 3, 22 (2017).

3. M.G. Kamalieva, R.A. Asrutdinova, S.M. Garipov, Influence of maintenance conditions of rearing young chickens on the formation of immunity and quality of meat, Bulletin of KrasSAU, 5, 35-39 (2017).

4. V.I. Fisinin, P. Surai, Immunity in modern livestock and poultry farming: from theory to practice of immunomodulation, Poultry, 5, 4-10 (2013).

5. E.A. Ovseychik, Cultivation of broiler chicken using immunomodulators, Poultry farming, 11-12, 41-42 (2018).

6. Yu.V. Krasnobaev, Astravit support of immunity in critical periods, Poultry farming, 4, 21-23 (2020).

7. S.V. Fedotov, M.N. Chernykh, E.A. Kapitonov, Application of immunomodulators for non-specific prevention of mono- and mixed infections in chickens, Bulletin of Altai SAU, 5, 97-103 (2012).

8. A.V. Sanin, A.A. Videnina, A.N. Narovlyansky et al., Use of immunomodulators in poultry farming, Poultry and poultry products, 1, 45 (2012).

9. A.S. Kuznetsov, A.S. Ushakov, Application of antibacterial immunostimulating additive in the composition of feed for broiler chickens, Poultry farming, 11, 44-47 (2020).

10. R.A. Asrutdinova, Results of using some immunomodulators to increase calf resistance, Scientific notes of the Bauman Kazan State Academy of Veterinary Medicine, 211, 214-218 (2012).

11. I.F. Barinsky, A.A. Lazarenko, L.M. Alimbarova, Study of the effectiveness of domestic immunomodulators, as well as their combined action with specific vaccines in experimental arbovirus infections, Immunology, 4, 181-183 (2012).

12. K.V. Zimin, Monosporin probiotic as a stimulator of the humoral arm of the immune response of the body of animals and birds to bacterial infections, Poultry and poultry products, 2, 50 (2016).

13. E.S. Voronin, A.M. Petrov, M.M. Serykh, Immunology (Moscow, 2002).

14. C.L. Hofkar, Prevention of immunodeficiency through vaccination program, BIO, 11, 2-4 (2003).

15. M.E. Dmitrieva, E.D. Dzhavadov, Features of vaccine prevention of immunosuppressive diseases of birds in industrial poultry farming, Farm animals, 3, 81-83 (2013).

16. E.D. Dzhavadov, M.E. Dmitrieva, Features of vaccine prevention in industrial poultry farming, Poultry and poultry products, 5, 37-39 (2011).

17. L.A. Vengerenko, Veterinary problems of poultry farming and their solution, Veterinary of farm animals, 3, 3-8 (2007).

18. Y. Gao et al., Effects of water-soluble Ganodermalucidum polysaccharides on the immune functions of patients with advanced lung cancer, J. Med. Food, 2, 159-168 (2005).

19. E.A. Laksaeva, I.A. Sychev, Influence of watersoluble polysaccharide complex of garden serviceberry on morphophysiological and biochemical parameters of laboratory rats, Russian Medical and Biological Bulletin named after Academician I.P. Pavlov, 2, 58-64 (2015).

20. I.A. Sychev, Influence of Melilot polysaccharide yellow pectin on some properties of the animal immune system, Russian Medical and Biological Bulletin named after Academician I.P. Pavlov, 1-2, 75-82 (2004). 